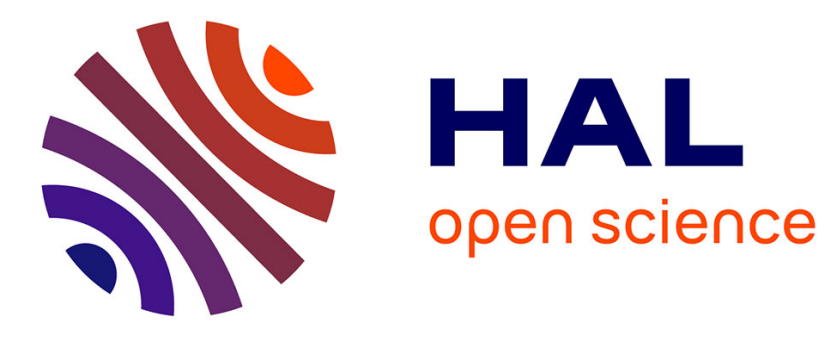

\title{
Statistical vibration-based damage localization on Saint-Nazaire Bridge mockup
}

Md Delwar Hossain Bhuyan, Yann Lecieux, Jean-Christophe Thomas, Cyril Lupi, Franck Schoefs, Michael Döhler, Laurent Mevel

\section{- To cite this version:}

Md Delwar Hossain Bhuyan, Yann Lecieux, Jean-Christophe Thomas, Cyril Lupi, Franck Schoefs, et al.. Statistical vibration-based damage localization on Saint-Nazaire Bridge mockup. 2018 - 40th IABSE Symposium, Sep 2018, Nantes, France. pp.1-8. hal-01886656

\section{HAL Id: hal-01886656 \\ https://hal.inria.fr/hal-01886656}

Submitted on 3 Oct 2018

HAL is a multi-disciplinary open access archive for the deposit and dissemination of scientific research documents, whether they are published or not. The documents may come from teaching and research institutions in France or abroad, or from public or private research centers.
L'archive ouverte pluridisciplinaire HAL, est destinée au dépôt et à la diffusion de documents scientifiques de niveau recherche, publiés ou non, émanant des établissements d'enseignement et de recherche français ou étrangers, des laboratoires publics ou privés. 


\title{
Statistical vibration-based damage localization on Saint-Nazaire Bridge mockup
}

\author{
Md Delwar Hossain Bhuyan
}

BAM Federal Institute for Materials Research and Testing, Berlin, Germany

Yann Lecieux, Jean-Christophe Thomas, Cyril Lupi, Franck Schoefs

GeM, Nantes University, Nantes, France

Michael Döhler, Laurent Mevel

Univ. Rennes, Inria/IFSTTAR, Rennes, France

Contact: michael.doehler@inria.fr

\begin{abstract}
The characterization of damages from output-only vibration measurements is an important issue for Structural Health Monitoring (SHM), in particular for bridges under ambient excitation. In the last years, a multitude of methods has been proposed for vibration-based damage detection, localization and quantification. In this work, a benchmark application for such methods is proposed, namely a $1 / 200$ scale model of the Saint-Nazaire Bridge, which is a cable-stayed bridge spanning the Loire River near the river's mouth. The region of interest, the central metallic structure, measures 720 meters. The aim of the instrumentation is to assess the capability of damage assessment methods to assess a cable failure. The model is instrumented with ten accelerometers and excited by white noise. A damage localization method is applied to test the proposed setup, namely the statistical damage locating vector approach (S-SDDLV). With this method, vibration measurements from the (healthy) reference and damaged states of the structure are confronted to a finite element of the reference state. Damage indicators are provided for the different structural elements that are easy to compute, without updating the model parameters, and taking into account the intrinsic uncertainty of noisy measurements.
\end{abstract}

Keywords: cable-stayed bridge; cable failure; vibration measurements; damage localization; structural health monitoring

\section{Introduction}

Structural Health Monitoring (SHM) aims at observing the physical parameters of a structure based on sensor measurements distributed at critical points of the structure. The measurement chain aims in many cases at the detection and localization of damages from data coming from vibration measurements. Vibration-based methods for damage assessment have been developed 
intensively in the last decades [1,2]. Hereby, methods for damage detection are the most developed since they can operate purely data-based and do not require a finite element (FE) model of the monitored structure. Automated methods for the next level of damage diagnostic, damage localization, are more sophisticated since a link between the measurement data and the physical properties of the structure is required, which is often given by a FE model. In this paper, the statistical damage locating vector approach (S-SDDLV) [3-6] is applied. It is based on data-driven features from data of the reference and damaged states, which are confronted to a FE model of the investigated structure to define statistical damage indicators for the structural elements, instead of updating the FE model. In this way, the requirements on the accuracy of the model are less strict and possible ill-posedness of FE updating is avoided.

This topic has generated a great deal of work related to the development of methods. On the other hand, few experimental works applied to complex structures (more complex than beams) are available. Experimental works are focused on the study of two categories of structures: models tested in the laboratory, or real structures damaged and tested before destruction. A lab benchmark that has been evaluated by several research groups is e.g. the ASCE Benchmark Test Frame at the University of British Columbia in Vancouver, Canada, which was established in 2002 [7]. Recent ambient vibration tests have been performed on the structure in 2016 [8]. Further lab structures (truss, tower, cable-stayed bridge model) are reported in [9]. An important example of a field test is the progressive damage test on Z24 Bridge in 1998, which was a post-tensioned concrete twocell box girder bridge in Switzerland [10]. More recently, a progressive damage test has been performed on S101 Bridge in 2008, which was a prestressed concrete bridge in Austria [11].

The objective of this study is to propose a benchmark for damage assessment on a cable-stayed bridge structure using vibration data only. The experimental work was carried out in the lab on a model scale $1 / 200$ of the bridge of Saint-Nazaire in France, equipped with accelerometers. The damage to identify is the rupture of one of the cables supporting the deck (see some examples in [12]).

\section{Material: Saint-Nazaire Bridge mock-up}

\subsection{The real structure}

The Saint-Nazaire Bridge is a cable-stayed bridge spanning the Loire River near the river's mouth in the west of France. The bridge includes also two access viaducts supported by pile foundations (Figure 1). The northern viaduct (1115 m long), and the southern one (1521 m long) are made of prestressed concrete. The pile foundations of the central part are also made of concrete. The main structure (in blue on Figure 1) is composed of a $720 \mathrm{~m}$ long cable-stayed metallic frame. The bridge deck, the cables and the triangular bridge pylons are made of steel.

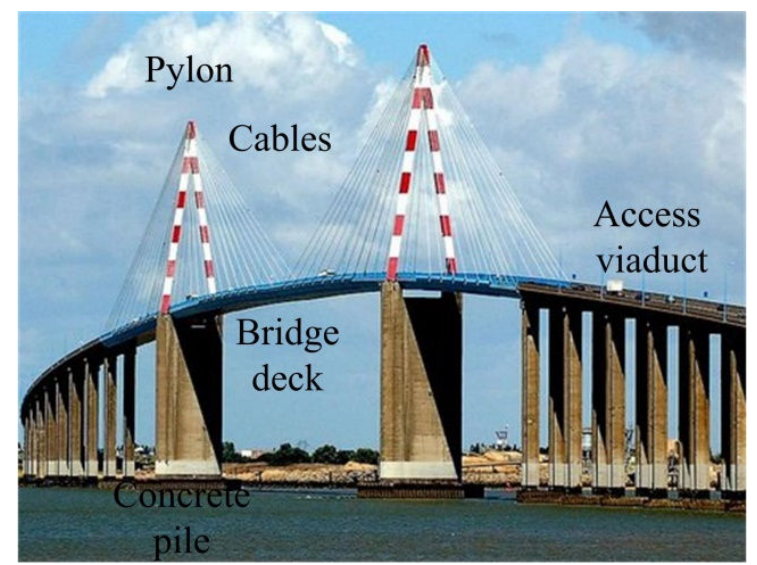

Figure 1. View of Saint-Nazaire Bridge

The concrete components were built by the company Société Générale d'Entreprises while the metallic components where built by the Compagnie Française d'Entreprises Métalliques. The construction was completed in 1974 and the structure was delivered in 1975. At the time of its construction it was the longest cable-stayed bridge in Europe. This bridge is a strategic structure, used by 30,000 vehicles a day, regularly maintained to ensure its accessibility. It is exposed to difficult climatic conditions, such as gusts of wind or storms. Signal lights placed at the ends of the bridge prohibit its crossing when the anemometer located in the middle of the deck on the west side detects a wind speed greater than 120 $\mathrm{km} / \mathrm{h}$. Some keys figures are listed below to describe the structure: 
- 72 cables

- 56 concrete piles

- Total length: 3356 meters

- Central structure: 720 meters

- Central span: 404 meters

- Height of deck of central bridge: 68 meters

- Height of the two central pylons: 130 meters

- Air draft of 61 meters

\subsection{The mockup}

For the dynamic study of the structure, only the central bridge (metallic part) was considered. The study presented here was carried out on a $1 / 200$ scale model of the structure, i.e. a $3.6 \mathrm{~m}$ long mockup positioned on two surface plates (Figure 2). To obtain the geometrical and mechanical characteristics of the model, the length of the real structure is multiplied by a scale factor of $1 / 200$. In order to preserve the scale factor for the masses, it was chosen to use building materials for the model with density similar or close to those used for the construction of the real bridge.

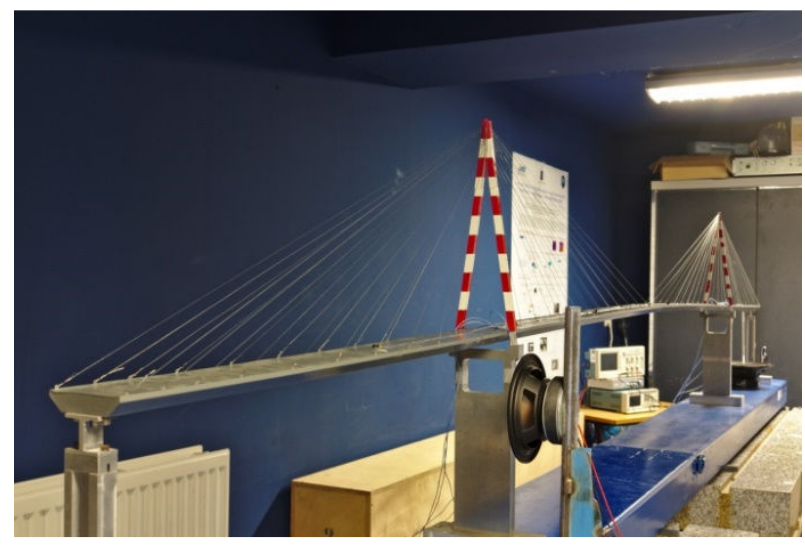

Figure 2. Saint Nazaire bridge mockup

For reasons of confidentiality on the characteristics of the real structure or for reasons of manufacturing constraints of the model, the model masses and geometry may slightly differ for the data computed using the scale factor of $1 / 200$, especially the mass of the bridge deck and the diameter of the cables. Thus, we list only the values actually measured on the final mock-up.

- Bridge pylons: they are in steel, with sections of dimension $4 \mathrm{~mm} \times 6 \mathrm{~mm}$ for the bottom parts. The two triangular parts are assembled on the upper part made with steel. The section dimensions are $10 \mathrm{~mm} \times 2 \mathrm{~mm}$. This part is drilled to fix the cables (Figure 3 ).

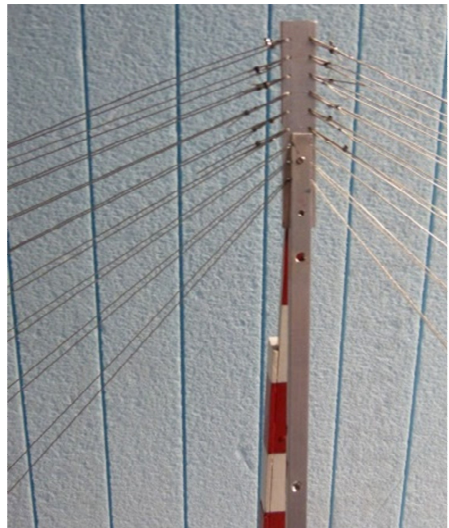

Figure 3. Detailed view of the mock-up bridge pylons and cable

- Cables: The cables are in steel. On the actual bridge, the section diameter is variable according to the length of the cables. In the model, all the cables are of identical section of $0.38 \mathrm{~mm}$ in diameter.

- Concrete piles: their dimensions are exactly those of the actual bridge piles (scale 1/200). They are built in aluminium since this material has a density very close to that of concrete (Concrete $\rho=2500 \mathrm{~kg} / \mathrm{m} 3$, aluminium $\rho=2700$ $\left.\mathrm{kg} / \mathrm{m}^{3}\right)$.

- Bridge deck: the actual deck is a steel structure whose section is empty. It is made of several different sections but whose geometric properties are close. For the model, an identical section at any point of the deck is considered. It is made from a $0.1 \mathrm{~mm}$ thick steel sheet. The actual section is complex and consists of different parts of metal reinforcements that limit the deformation of the section. For the model, this role is provided by a light Young Modulus Rohacell foam ( $E=$ $74 \mathrm{MPa}$ and $\rho=51 \mathrm{~kg} / \mathrm{m}^{3}$ ). The foam and the steel sheet are glued together using an epoxy adhesive.

\subsection{Acquisition and excitation devices}

On the bridge model, there are 10 miniature piezoelectric accelerometers $(0.8 \mathrm{gm})$ of sensitivity $100 \mathrm{mV} / \mathrm{g}$ (PCB of reference ICP) capable of measuring vertical accelerations. All the signals are collected on a data-logger (HBM reference MX16101) at the acquisition frequency of $4800 \mathrm{~Hz}$. The excitation is provided by an audio boomer (see Figure 4) which acts as a shaker. It is powered 
by the signal of a white noise generator $(+/-5 \mathrm{~V}$ peak to peak, manufactured by Tektronix, reference AFG), amplified by a stereo amplifier. The signal is controlled with an oscilloscope. The devices of the signal acquisition chain and the shaker are visible in Figure 4.

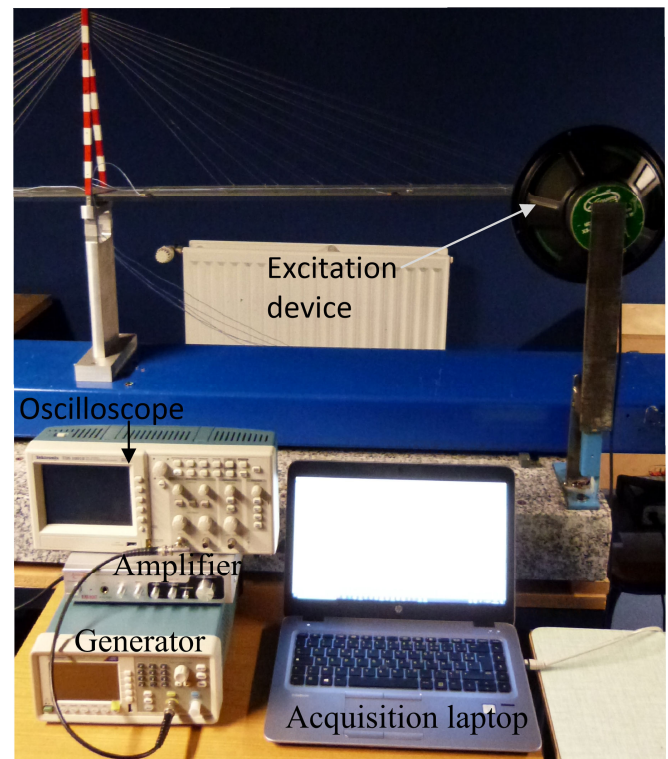

Figure 4. Acquisition and excitation devices

\subsection{Damage to structure}

The damage to assess is a failure of a cable supporting the deck. Physically, the rupture is simulated by removing the fastener connecting the cable and the deck. Thus, only the stiffness of the structure is modified and not its mass. Two damages are tested, the rupture of a cable, and the rupture of two cables symmetrical with respect to the pylon. These two elements located in the middle of the bridge are visible in Figure 5.

\section{Method}

\subsection{The Saint Nazaire bridge mock-up finite element model}

The goal is to make available the experimental data as well as the numerical model. With this in mind, it was decided to propose a finite element modeling compatible with the student version of the ABAQUS software (available online and downloadable free of charge). This software also offers the possibility of dialogue with other software (including MATLAB) through its Python interface. The mock-up is modeled only with beam elements for pylons, deck and bridge piers while truss elements are used for the cables modelization (see Figure 5). Each cable is discretized with a unique truss finite element. The damaged elements are numbered 37 and 38 .

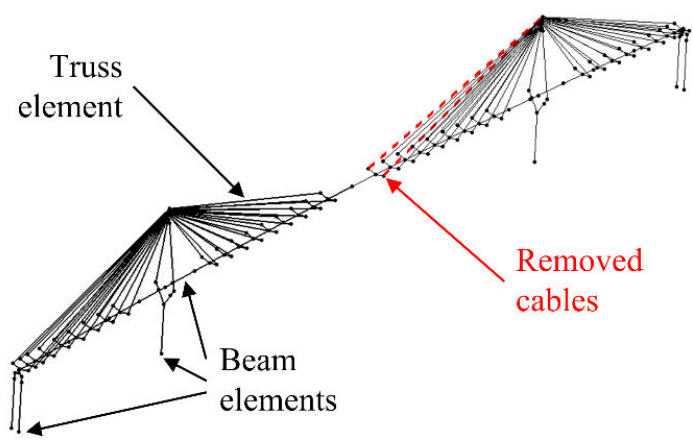

Figure 5. Saint-Nazaire bridge mockup finite element model

The material law considered here is linear elasticity only. Geometric nonlinearities are not taken into account. The behavior model of the bridge is therefore totally linear.

The sections measured on the model have been associated with the different finite elements of the bridge as long as they are classical forms (rectangular or circular section). The material parameters (Young's modulus and Poisson's ratio) are those derived from classical charts. For the deck, on the other hand, it was chosen to describe the section by giving the values of the section, the linear density and the quadratic moments in bending and in torsion. These were evaluated by static loading tests carried out on the deck alone (without cables). The finite element model is based only on these parameters. No updating of the model parameters based on the data resulting from the vibration analysis was performed. The finite element model consists of 180 beam elements and 72 truss elements for a total of 1062 degrees of freedom.

\subsection{Damage localization with S-SDDLV method}

To test the capabilities of the test structure for damage localization, the statistical extension of the Stochastic Dynamic Damage Locating Vector (S-SDDLV) approach has been applied. The SDDLV is an output-only damage localization method 
based on interrogating changes $\delta G(s)$ in the transfer matrix $G(s)$ of a system in both reference and damaged states [3], $s$ is a Laplace variable in the complex plane. A vector is obtained in the null space of $\delta G(s)$ from system identification results using output-only measurements corresponding to both states. Then this load vector is applied to the Finite Element (FE) model of the structure for the computation of a stress field over the structure. Damage localization is related to this stress field where the computed stress is zero or close to zero in practice [3]. In this section, the underlying computation of the stress field and its statistical evaluation is summarized.

\subsubsection{Models}

The behavior of a mechanical structure is assumed to be described by a linear time-invariant (LTI) dynamic system

$$
\mathbf{M} \ddot{z}(t)+\mathbf{C} \dot{z}(t)+\mathbf{K} z(t)=f(t)
$$

where $\mathbf{M}, \mathbf{C}, \mathbf{K} \in \mathbb{R}^{d \times d}$ are the mass, damping and stiffness matrices, respectively, $t$ indicates continuous time and $z \in \mathbb{R}^{d}$ the displacements at the $d$ degrees of freedom (DOF) of the structure. The external force $f(t)$ is not measurable. Observing system (1) at $r$ sensor coordinates, it can be transformed to the corresponding continuous-time state-space model [3]

$$
\left\{\begin{array}{l}
\dot{x}(t)=A x(t)+B e(t) \\
y(t)=C x(t)+D e(t)
\end{array}\right.
$$

with state $x \in \mathbb{R}^{n}$, output $y \in \mathbb{R}^{r}$, state transition matrix $A \in \mathbb{R}^{n \times n}$ and output matrix $C \in \mathbb{R}^{r \times n}$, where $n=2 d$ is the system order and $r$ is the number of outputs. The input influence and direct transmission matrices are $B \in \mathbb{R}^{r \times n}$ and $D \in \mathbb{R}^{r \times r}$ respectively. Matrices $(A, C)$ contain the information on the modal parameters of the structure and can be identified from measurements.

\subsubsection{Computation of damage indicator}

The transfer matrix $G(s)$ of system (2) writes

$$
G(s)=R(s) D,
$$

where $R(s)=C(s I-A)^{-1}\left[\begin{array}{c}C A \\ C\end{array}\right]^{\dagger}\left[\begin{array}{l}I \\ 0\end{array}\right][3], I$ is the identity matrix of size $r, 0$ is zero matrix, and $\dagger$ denotes the Moore-Penrose pseudoinverse. The difference of the transfer matrices in both damaged (using tilde superscript) and healthy states is $\delta G(s)=\tilde{G}(s)-G(s)$. The matrices $\delta G(s)$ and $\delta R^{T}(s)=\tilde{R}^{T}(s)-R^{T}(s)$ have the same null space [2], which is obtained from the Singular Value Decomposition (SVD)

$$
\delta R^{T}(s)=U \Sigma V^{H}=\left[\begin{array}{ll}
U_{1} & U_{2}
\end{array}\right]\left[\begin{array}{cc}
\Sigma_{1} & 0 \\
0 & \Sigma_{2}
\end{array}\right]\left[\begin{array}{ll}
V_{1} & V_{2}
\end{array}\right]^{H}
$$

where $U, \Sigma, V \in \mathbb{C}^{r \times r}, \Sigma_{2} \approx 0$ and $H$ indicates the conjugate transpose. The desired load vector $v(s)$ is obtained from $V_{2}$, e.g. as the last column. Note that only output data is necessary for the computation of estimates of the system matrices $A$ and $C$ in both reference and damaged states, e.g. through stochastic subspace identification (SSI) [13], and subsequently of an estimate of $v(s)$. To compute the stress field, the load vector $v(s)$ is applied to the reference FE model of the structure. This stress $S(s)$ yields a linear relation to the load and can thus be expressed through a matrix multiplication

$$
S(s)=L_{\text {model }}(s) v(s)
$$

where $L_{\text {model }} \in \mathbb{C}^{l \times r}$ stems from the FE model of the structure [4-6]. Theoretically, the entries in the stress vector $S(s)$ that are zero indicate potential damage in the corresponding structural elements. However, these stresses are not exactly zero but small in practice because of modal truncation, model errors and estimation uncertainties due to noisy measurements. To decide if a structural element is potentially damaged, the corresponding stress components are tested for being zero in a statistical test. The covariance of the stress $\Sigma_{S}$ has been estimated in [4-6]. Then, an appropriate test is performed on each structural element $t$ by selecting the respective stress components $S_{t}$ in $S(s)$ as well as the covariance submatrix $\Sigma_{t}$ of $\Sigma_{S}$ and computing the test statistic

$$
\chi_{t}^{2}=S_{t}^{T} \Sigma_{t}^{-1} S_{t}
$$

for each structural element $t$ tested for damage. Since stress over damaged elements is zero in theory, potential damage is located in elements $t$ corresponding to the lowest values of $\chi_{t}^{2}$ among all elements. Robustness of the localization is achieved by evaluating the stress in (6) for different Laplace variables $s$ when computing the null space vector $v(s)$ and aggregating the results for each element $[5,6]$. 


\section{Results and discussion}

Ten sensors are located on the structure in the vertical direction. For damaged and healthy states, acceleration data containing 581,118 and 588,620 samples, respectively, at a sampling frequency of $4800 \mathrm{~Hz}$ were recorded. The data was low-pass filtered and downsampled by factor 6 . As a reminder, damage is introduced by removing cables number 37 and 38 at the middle of the structure.

\subsection{Modal analysis}

In the first step, the modes of the structure were identified from the measurements in both healthy and damaged states using covariance-driven subspace identification, and then compared to the modes present in the model. The first four vertical bending modes were well estimated in both states together with their uncertainty information. The respective stabilization diagram on a dataset from the healthy state is shown in Figure 6 and the identified frequencies are shown in Table 1 for each mode. Note that while more than four modes are present in the stabilization diagram, we concentrate the analysis only on the first four since the higher modes were badly estimated or did not match well with the FE model.

The identified mode shapes of the first four modes are shown in Figures 8 and 9 for the healthy and damaged states, respectively, together with the mode shapes obtained from the FE model in the healthy state.

To check the accordance of the identified modes with the FE model, a MAC diagram (Modal Assurance Criteria) is computed on shown in Figure 10, where the MAC values between the four identified modes from the data in the healthy state vs. the modes from the FE model are illustrated.

Comparing the identified frequencies to those of the FE model, it turns out that there is some discrepancy (see Table 2). Similarly, the identified mode shapes show some differences (Figure 8). It should be noted that the damage localization method does not require a perfect match between data and model in the reference state, which is an important property of the method for its application in practice. Instead, it only needs to be verified that the stress computation is accurate in relative terms between the different structural elements (up to a constant factor), which requires less accuracy of the FE model than for classical updating based methods.

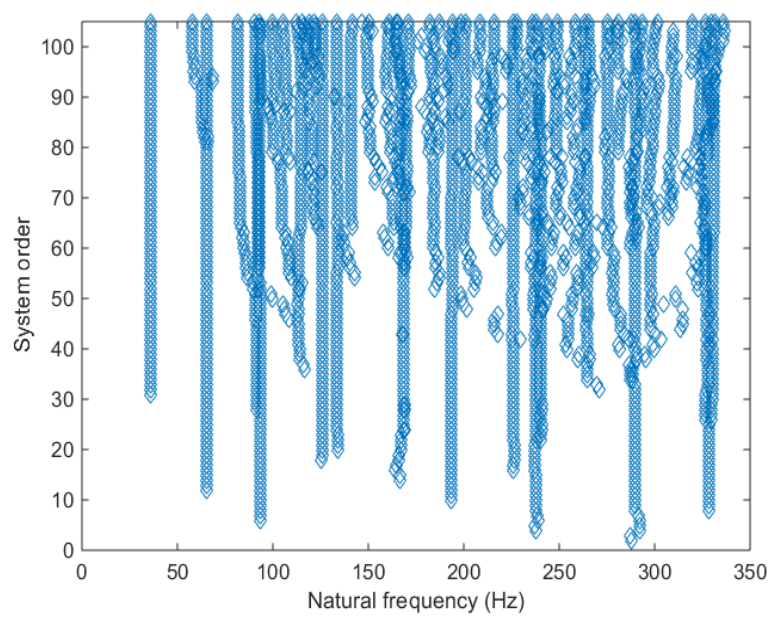

Figure 6. Stabilization diagram from dataset in the healthy state using SSI

Table 1. Identified frequency (f $[\mathrm{Hz}])$ and damping ratio ( $\xi[\%])$ from data

\begin{tabular}{ccccc}
\hline \multirow{2}{*}{ Mode } & \multicolumn{2}{c}{ Healthy state } & \multicolumn{2}{c}{ Damaged state } \\
\cline { 2 - 5 } & $f$ & $\xi$ & $f$ & $\xi$ \\
\hline 1 & 36.2 & 1.5 & 35.5 & 1.9 \\
\hline 2 & 65.5 & 1.4 & 64.6 & 0.9 \\
\hline 3 & 92.7 & 0.9 & 91.5 & 3.3 \\
\hline 4 & 133.7 & 1.6 & 132.2 & 0.6 \\
\hline
\end{tabular}

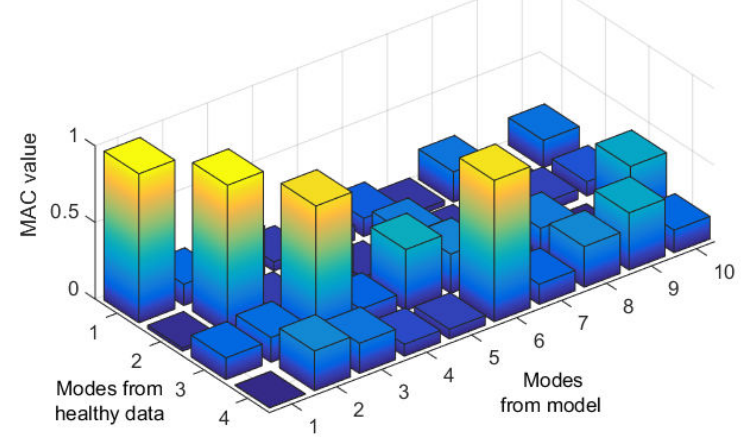

Figure 10. MAC diagram between identified modes from data and vertical modes from FE model

Table 2. Comparison of the frequency $(\mathrm{Hz})$ from model and data from healthy state

\begin{tabular}{ccc}
\hline Mode & $\boldsymbol{f}$ (model) & $\boldsymbol{f}$ (data) \\
\hline 1 & 35.9 & 36.2 \\
\hline 2 & 61.1 & 65.5 \\
\hline 3 & 88.6 & 92.7 \\
\hline 4 & 121.5 & 133.7 \\
\hline
\end{tabular}




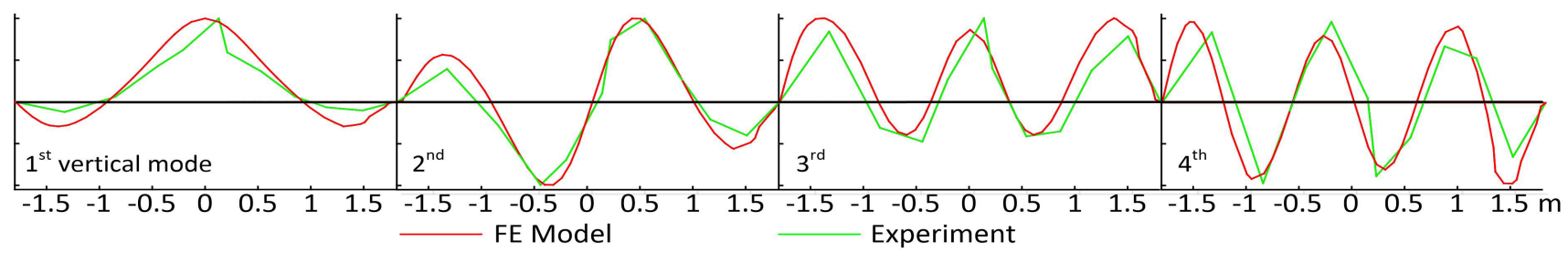

Figure 8. Experimental mode shapes (green) of the first four modes in the healthy state and mode shapes from FE model in healthy state (red).

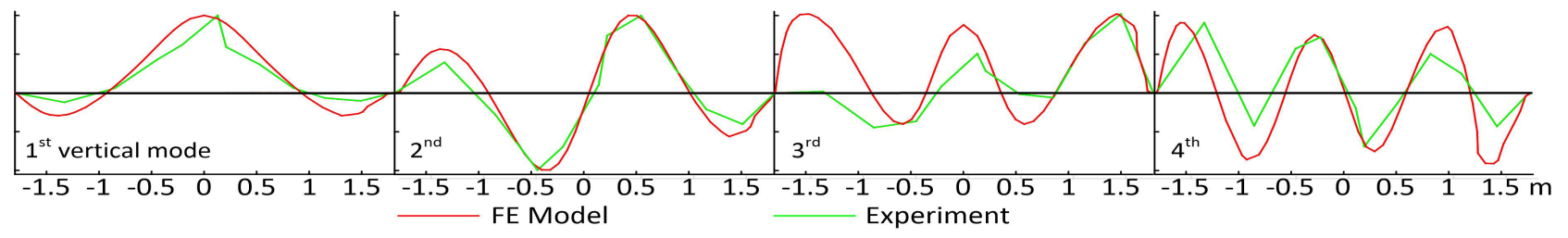

Figure 9. Experimental mode shapes of the first four modes in the damaged state (green) and mode shapes from FE model in healthy state (red).

\subsection{Damage localization results}

The localization results are obtained for all 72 cables based on the stress computation and its covariance for the statistical evaluation in the $\chi_{t}^{2}$ tests. They are carried out for three different choices of $s$ Laplace variables, each in the vicinity of the identified modes. First, one $s$ Laplace variable is chosen at $s_{1}=-1+300 i$; second, two $s$ Laplace variables are chosen at $s_{1}=-1+300 i$ and $s_{2}=-1+400 i$, and third, four $s$ - Laplace variables are chosen at $s_{1}=-1+300 i, s_{2}=$ $-1+400 i, s_{3}=-1+380 i$ and $s_{4}=-1+760 i$ for joint evaluation. To compare the magnitude between the healthy and damaged elements, the computed values are normalized such that the smallest value is 1 . The estimated stresses and their statistical evaluation are shown in Figures 11(a) and 11(b)-(d), respectively. It can be seen that damage is correctly localized at elements 37 and 38 . Notice that the ratio between undamaged and damaged elements is higher in the statistical evaluation in Figure 11(b), compared to the deterministic stress computation in Figure 11(a). By adding more information using 1 and 3 Laplace variables in the same setting, it can be seen that the contrast ratio to healthy elements is further increased in Figure 11(d).

Summarizing the localization results in this application, it can be seen that using both the estimated stress from the SDDLV approach and its statistical evaluation is sufficient for damage localization using four identified modes.

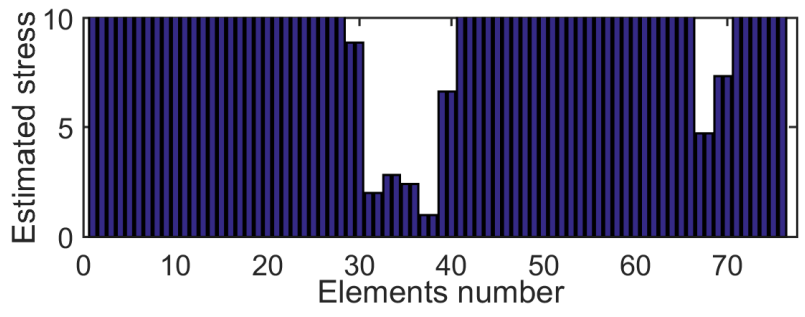

Figure 11(a). Estimated stress, one s-value

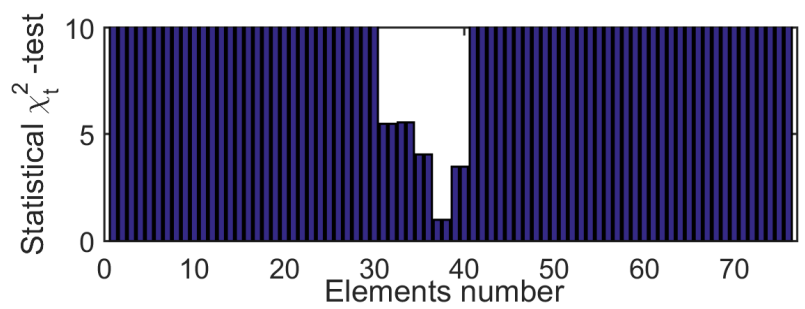

Figure 11(b). Statistical tests, one s-value

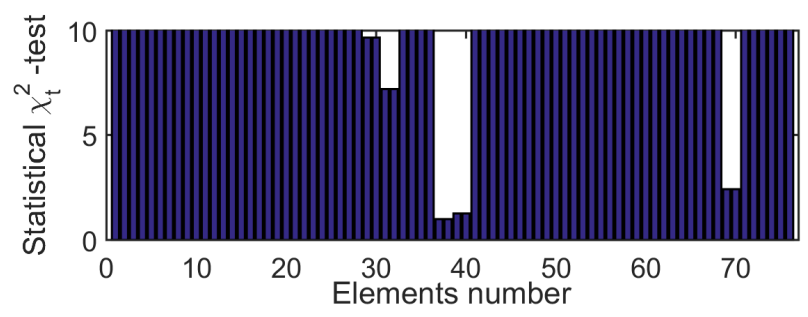

Figure 11(c). Statistical tests, two s-values

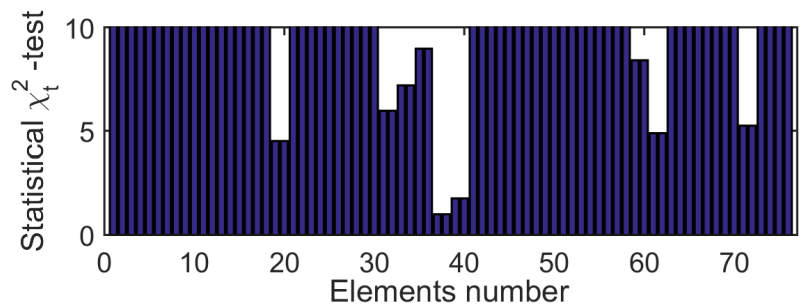

Figure 11(d). Statistical tests, four s-values 


\subsection{Discussion}

In this example, the damages could be correctly localized with the S-SDDLV method. Notice that the frequency shift between reference and damaged states is rather small (around 1\% decrease, Table 1). Moreover, the FE model of the structure was quite rough and the modes did not match perfectly in the reference state (see Table 2). This is an important feature for the application of the damage localization method in practical situations, where FE models are not perfect. Since the selected localization method is strongly based on the (FE model-based) stress evaluation for a particular load (that is calculated purely on the measurement data), but not directly on the parameters of the FE model, the requirements on the accuracy of the model are indeed less strong.

\section{Conclusion}

In this paper, a benchmark structure for vibrationbased damage assessment was presented and the introduced damage was correctly localized with one particular method, the S-SDDLV. Here, only one test case was used and one method was applied. The aim is to provide the experimental data for several test cases including damages in different cables and measurements in both vertical and horizontal directions, and to make the data and the FE model of the structure publicly available as a benchmark for the application of vibrationbased methods for damage assessment. These data should enable researchers to assess the performances of their damage identification methods on a complex structure but in a controlled environment. Indeed, the tests are performed in the lab and the only variable parameter is the rigidity of the structure. This test is therefore a possible validation step before applying the methods to real structures subject to temperature variations, thermal gradients or changes in mass over time.

\section{Acknowledgements}

Financial support from the Bretagne region is gratefully acknowledged. The authors thank the physics department of the University of Nantes for providing the reduced-scale bridge and the acquisition system.

\section{References}

[1] Carden E. and Fanning P. Vibration based condition monitoring: a review. Structural Health Monitoring 2004; 3(4): 355-377.

[2] Fan W. and Qiao P. Vibration-based damage identification methods: a review and comparative study. Structural Health Monitoring 2011; 10(1): 83-111.

[3] Bernal D. Load vectors for damage location in systems identified from operational loads. Journal of Engineering Mechanics, 2010; 136(1):31-39.

[4] Döhler M., Marin L., Bernal D., and Mevel L. Statistical decision making for damage localization with stochastic load vectors. Mechanical Systems and Signal Processing, 2013; 39(1-2):426-440.

[5] Marin L., Döhler M., Bernal D., and Mevel L. Robust statistical damage localization with stochastic load vectors. Structural Control and Health Monitoring, 2015; 22:557-573.

[6] Bhuyan M.D.H., Döhler M., Lecieux Y., Mevel L., and Schoefs F. Statistical damage localization with stochastic load vectors using multiple mode sets. Structural Health Monitoring, 2017; 16(5):518-535.

[7] Ventura C.E., Lord J.F., Turek M., Sereci A.M., Radulescu D., and Radulescu, C. Experimental studies and remote monitoring of IASC-ASCE benchmark test frame. In Proc. of the XXI International. Modal Analysis Conference (pp. 3-6); 2003.

[8] Allahdadian S., Döhler M., Ventura C., and Mevel L. Damage localization of a real structure using the statistical subspace damage localization method. In Proc. 11th International Workshop on Structural Health Monitoring; 2017.

[9] Zhou L., Yan G., Wang L., and Ou J. Review of benchmark studies and guidelines for structural health monitoring. Advances in Structural Engineering. 2013; 16(7):1187-1206.

[10] Reynders E., and De Roeck G. Continuous vibration monitoring and progressive damage testing on the Z24 bridge. In C. Boller, F.K. Chang, and Y. Fujino, editors, Encyclopedia of Structural Health Monitoring, John Wiley \& Sons, New York, NY, 2009.

[11] Döhler M., Hille F., Mevel L., and Rücker W. Structural health monitoring with statistical methods during progressive damage test of S101 Bridge. Engineering Structures. 2014; 69: 183-193.

[12] Nghia N.T. and Samec V. Cable stay bridges investigation of cable rupture. Journal of Civil Engineering and Architecture. 2016; 10:270-279.

[13] Döhler M., and Mevel L. Efficient multi-order uncertainty computation for stochastic subspace identification. Mechanical Systems and Signal Processing. 2013; 38(2):346-366. 